\title{
Evaluation of Leptomeningeal Contrast Enhancement Using Pre-and Postcontrast Subtraction 3D-FLAIR Imaging in Multiple Sclerosis
}

(D) Zivadinov, (DD.P. Ramasamy, (D). Hagemeier, (D). Kolb, (D) N. Bergsland, (D)F. Schweser, (D) M.G. Dwyer, (D)B. Weinstock-Guttman, and (DD. Hojnacki

\begin{abstract}
BACKGROUND AND PURPOSE: Leptomeningeal contrast enhancement is found in patients with multiple sclerosis, though reported rates have varied. The use of 3D-fluid-attenuated inversion recovery pre- and postcontrast subtraction imaging may more accurately determine the frequency of leptomeningeal contrast enhancement. The purpose of this study was to investigate the frequency of leptomeningeal contrast enhancement using the pre- and postcontrast subtraction approach and to evaluate 3 different methods of assessing the presence of leptomeningeal contrast enhancement.
\end{abstract}

MATERIALS AND METHODS: We enrolled 258 consecutive patients with MS (212 with relapsing-remitting MS, 32 with secondaryprogressive MS, and 14 with clinically isolated syndrome) who underwent both pre- and 10-minute postcontrast 3D-FLAIR sequences after a single dose of gadolinium injection on $3 T$ MR imaging. The analysis included leptomeningeal contrast-enhancement evaluation on 3D-FLAIR postcontrast images in native space (method A), on pre- and postcontrast 3D-FLAIR images in native space (method B), and on pre-/postcontrast 3D-FLAIR coregistered and subtracted images (method C, used as the criterion standard).

RESULTS: In total, 51 (19.7\%) patients with MS showed the presence of leptomeningeal contrast enhancement using method A; 39 (15.1\%), using method $\mathrm{B}$; and $39(15.1 \%)$, using method $C(P=.002)$. Compared with method $\mathrm{C}$ as the criterion standard, method $\mathrm{A}$ showed $89.8 \%$ sensitivity and $92.7 \%$ specificity, while method B showed $84.6 \%$ sensitivity and $97.3 \%$ specificity $(P<.001)$ at the patient level. Reproducibility was the highest using method $C$ ( $\kappa$ agreement, $r=088, P<.001$ ). The mean time to analyze the 3D-FLAIR images was significantly lower with method $C$ compared with methods $A$ and $B(P<.001)$.

CONCLUSIONS: 3D-FLAIR postcontrast imaging offers a sensitive method for detecting leptomeningeal contrast enhancement in patients with MS. However, the use of subtraction imaging helped avoid false-positive cases, decreased reading time, and increased the accuracy of leptomeningeal contrast-enhancement foci detection in a clinical routine.

ABBREVIATIONS: $\mathrm{CE}=$ contrast enhancement; $\mathrm{CIS}=$ clinically isolated syndrome; $\mathrm{Gd}$ = gadolinium; $\mathrm{LM}=$ leptomeningeal; $\mathrm{LV}=$ lesion volume; $\mathrm{NPV}=$ negative predictive value; $P P V=$ positive predictive value; $R R=$ relapsing-remitting; $S P=$ secondary-progressive

$\mathbf{P}$ ersistent leptomeningeal (LM) inflammation in the form of ectopic lymphoid follicle-like structures ${ }^{1-4}$ is associated with development of subpial cortical lesions in patients with multiple sclerosis. $^{2-8}$

Received September 13, 2017; accepted after revision November 28.

From the Department of Neurology (R.Z., D.P.R., J.H., N.B., F.S., M.G.D.), Buffalo Neuroimaging Analysis Center, Jacobs School of Medicine and Biomedical Sciences; Translational Imaging Center at Clinical Translational Science Institute (B.W.-G.); and Department of Neurology (R.Z., C.K., D.H.), Jacobs Comprehensive MS Treatment and Research Center, University at Buffalo, State University of New York, Buffalo, New York.

This work was funded by the internal funds of the Buffalo Neuroimaging Analysis Center, Department of Neurology, Jacobs School of Medicine and Biomedical Sciences, University at Buffalo, State University of New York.

Please address correspondence to Robert Zivadinov, MD, PhD, FAAN, FEAN, FANA, Buffalo Neuroimaging Analysis Center, Translational Imaging Center at Clinical Translational Science Institute, University at Buffalo, State University of New York, 100 High St, Buffalo, NY 14203; e-mail: rzivadinov@bnac.net
Use of 3D-fluid-attenuated-inversion recovery MR imaging acquired 10 minutes post-contrast administration of gadolinium (Gd) has been recently proposed as a novel in vivo potential biomarker for the detection of persistent LM inflammation. It has been hypothesized that trapped contrast enhancement (CE) within the subarachnoid space of patients with $\mathrm{MS}^{8-10}$ and subjects in their prodromal disease stage ${ }^{11}$ is indicative of LM inflammation and can be visualized by 3D-FLAIR postcontrast MR imaging.

However, at this time, to our knowledge, there are no currently available consensus guidelines regarding the most sensitive and specific approach for the detection of LM CE in patients with MS

Indicates article with supplemental on-line tables

Indicates article with supplemental on-line photos.

http://dx.doi.org/10.3174/ajnr.A5541 
in vivo, so imaging of LM CE cannot be translated immediately to the clinical routine. Given the uncertainty in the literature as to the frequency of LM CE in patients with MS with different field strengths, pulse sequences, Gd-based protocols, and analysis methods, ${ }^{9,10,12-14}$ there is an urgent need to determine LM CE prevalence using different validation techniques. In previous studies, the estimates of LM CE frequency ranged from $<1 \%$ to $90 \%$. For example, using 3T MR imaging, Absinta et $\mathrm{al}^{9}$ showed that LM CE was detected in 74 of 299 (25\%) examined patients with MS, compared with 1 of 37 (2.7\%) healthy subjects. Using 7T MR imaging, Harrison et al ${ }^{14}$ showed that 26 of 29 (90\%) participants with MS had at least 1 foci of LM CE, suggesting that LM CE detection may be more prevalent using ultra-high-field MR imaging, which is also more in line with findings from histopathologic studies. ${ }^{8}$

In previous LM CE imaging studies, detection of LM CE was performed on pre- and/or postcontrast native space images, not oriented and sliced in exactly the same way, which could complicate their comparison and increase the likelihood of detection of false-positive findings. ${ }^{9-13}$

The purpose of this study was to investigate the frequency of LM CE using the pre- and postcontrast 3D-FLAIR subtraction approach and to evaluate 3 different methods of assessing the presence of LM CE in consecutively enrolled patients with MS consisting of relapsing-remitting $(\mathrm{RR})$ and secondary-progressive (SP) MS disease subtypes and in patients with clinically isolated syndrome (CIS) who were recruited in a clinical routine.

\section{MATERIALS AND METHODS Study Population}

The study consisted of 258 consecutive patients with MS (212 with RRMS, 32 with SPMS, and 14 with CIS) who were enrolled and underwent both pre- and 10-minute postcontrast 3D-FLAIR sequences after a single dose of Gd injection on 3T MR imaging.

The inclusion criteria for this retrospective study of LM CE in MS were the following: 1) patients with CIS, RRMS, or SPMS; 2) 18-75 years of age; 3 ) undergoing pre- and postcontrast 3DFLAIR sequences 10 minutes after a single dose of Gd injection using a standardized 3T MR imaging protocol; and 4) physical/ neurologic examination within 30 days from MR imaging. Exclusion criteria were the following: 1) the presence of relapse and steroid treatment within the 30 days preceding study entry, 2) pre-existing medical conditions known to be associated with brain pathology (cerebrovascular disease, positive history of substance abuse), or 3) pregnancy.

All subjects were assessed with physical and neurologic examinations. The study data collection was approved by the local institutional review board of the University at Buffalo.

\section{MR Imaging Acquisition and Analysis}

Subjects were examined with a 3T Signa Excite HD 12.0 scanner (GE Healthcare, Milwaukee, Wisconsin) using an 8-channel head and neck coil that did not undergo major hardware or software upgrades during the study.

The presence and frequency of LM CE were assessed using a 3D-FLAIR for enhanced lesion detection (3D-FLAIRED) sequence. $^{10,15}$ The pre- and post-3D-FLAIR sequences were ac- quired before and 10 minutes after an intravenous bolus of 0.1 $\mathrm{mmol} / \mathrm{kg}$ of gadolinium-DTPA injection in a sagittal acquisition with TR/TI/TE $=9000 / 2420 / 600 \mathrm{~ms}$, acquisition matrix $=256 \times$ 192 , voxel size $=1.0 \times 1.3 \times 1.3 \mathrm{~mm}^{3}$, frequency direction $=$ inferior to superior. ${ }^{16}$ The sequence uses variable flip angles so that the effective TE of $600 \mathrm{~ms}$ for the sequence was equal to a TE of $110 \mathrm{~ms}$ for a conventional spin-echo sequence ${ }^{10,15}$ and was 12 minutes 22 seconds long. A spin-echo 2D-T1WI was acquired using a $256 \times 192$ matrix and $256 \times 192 \mathrm{~mm}^{2} \mathrm{FOV}$, resulting in a nominal in-plane resolution of $1 \times 1 \mathrm{~mm}^{2}$ and 48 gapless 3 -mmthick slices with TE/TR $=16 / 600 \mathrm{~ms}$ before and 5 minutes after Gd injection, for detection of T1 hypointense and hyperintense (Gd) lesions, and each was 4 minutes 34 seconds long.

The 3D-FLAIR postcontrast image was rigidly registered to the 3D-FLAIR precontrast image. ${ }^{17}$ The $3 \mathrm{D}$-FLAIR precontrast image was then voxelwise subtracted from the coregistered 3D-FLAIR postcontrast image, yielding a subtraction map, which was subsequently smoothed with a Gaussian kernel of $0.5 \mathrm{~mm}$ (Figure).

MR imaging analysts were blinded to the subject's cohort and physical and neurologic conditions. The images were examined first on 3D-FLAIR postcontrast images in native space (method A, On-line Fig 1), pre- and postcontrast 3D-FLAIR images in native space (method B, On-line Fig 1), and pre/postcontrast 3D-FLAIR coregistered and subtracted images (method C, Figure) in a randomized manner to avoid potential practice effects. A 4-week delay was introduced for all 3 methods to further reduce potential recall bias. Additional analysis was conducted a posteriori to examine the concordance of all 3 methods. The LM CE foci were compared side by side in an unblinded manner to determine discordant cases (true-positive, true-negative, false-positive, and false-negative), determined by methods A and B compared with method C.

The LM CE analysis was performed by 2 experienced neuroimagers (D.P.R. and D.H. with $>15$ years of reading experience) fully blinded to other imaging modalities of the individual subject examinations. An additional expert neuroimager (R.Z. with $>15$ years of reading experience) served on a panel to reach a consensus when there were discrepancies by the 2 readers. The unblinded comparison was performed by a consensus of all 3 readers. The mean time to analyze the 3D-FLAIR images for the 3 methods included only the operator time for inspection of the images.

The reproducibility for detection of LM CE was obtained by 2 raters in 30 patients with MS for methods $\mathrm{A}, \mathrm{B}$, and $\mathrm{C}$, using a similar approach as previously reported. ${ }^{10}$

LM CE foci were defined as signal intensity within the subarachnoid space that was substantially greater than that of brain parenchyma. The images were reviewed using Jim software (http://www.xinapse.com/home.php) in the sagittal plane of the original acquisition and in additional coronal and axial views (On-line Fig 1). LM CE was evaluated according to aspect (focal or diffuse), location (within a sulcus or overlying the brain convexity, along a dural fissure, or traversing several of these areas), shape (nodular, linear, or platelike), cerebral hemisphere (right or left), tentorium (supra- or infratentorial), and lobe (frontal, parietal, temporal, and occipital) as previously reported..$^{9,10}$

T2-, T1-, and Gd lesion volume (LV) and number were calculated using a reliable semiautomated edge-detection contouring/ 


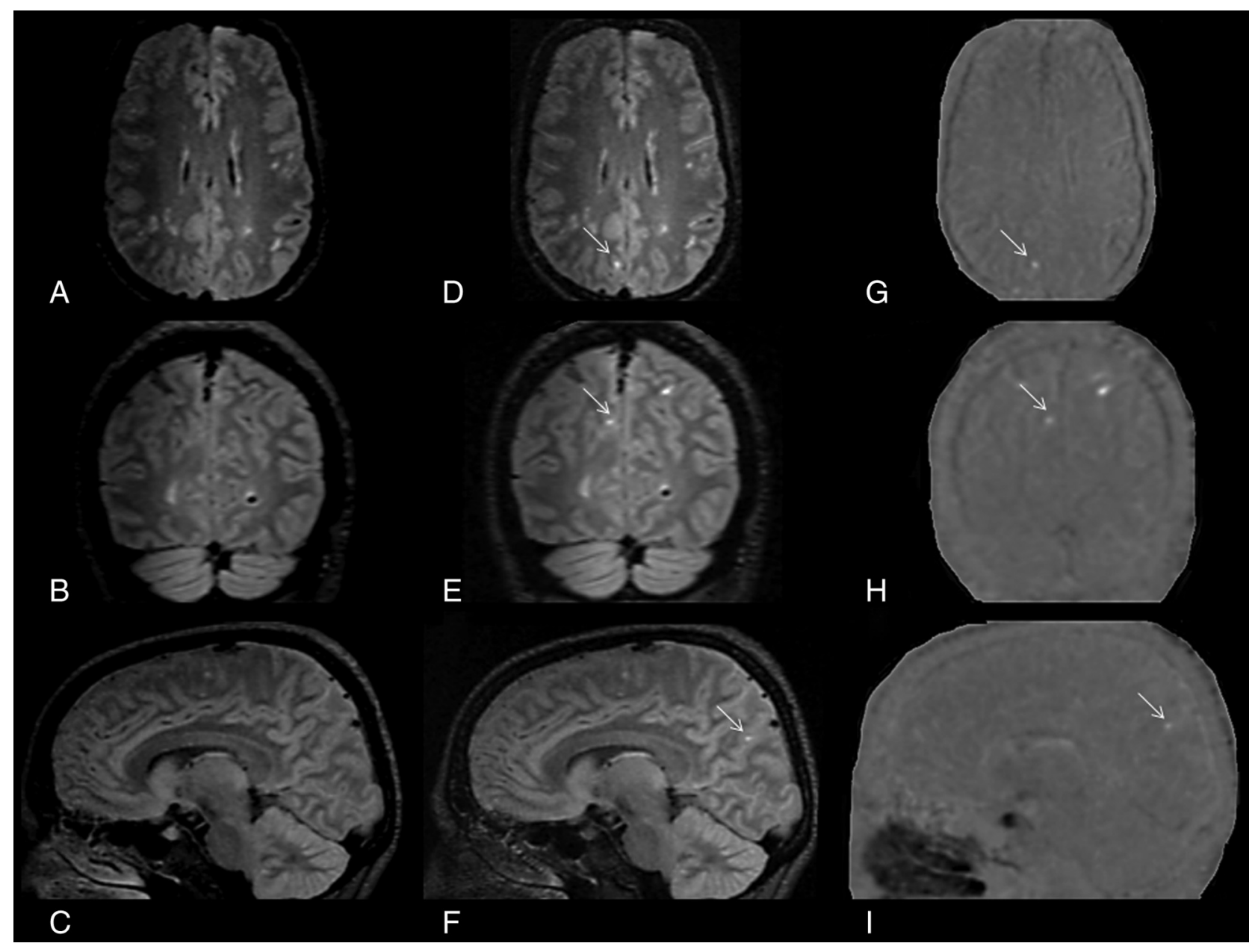

FIGURE. Detection of leptomeningeal contrast-enhancement foci using subtraction images. A-C, Coregistered 3D-FLAIR precontrast images in all 3 orthogonal planes. D-F, The corresponding coregistered 3D-FLAIR postcontrast images in all 3 orthogonal planes. G-I, The corresponding pre-/postcontrast 3D-FLAIR subtraction images in all 3 orthogonal planes. A patient with relapsing-remitting multiple sclerosis has a true LM CE in the right parietal region that was easily spotted with the aid of pre-/postcontrast 3D-FLAIR subtraction images, which otherwise would have been undetected.

thresholding technique on 3D-FLAIR and 2D-T1 pre- and postcontrast examinations, respectively. ${ }^{18}$

\section{Statistical Analysis}

Statistical analysis was performed using the Statistical Package for the Social Sciences, Version 21.0 (IBM, Armonk, New York). Differences in the categoric variables between the groups were analyzed using the $\chi^{2}$ test. Differences in clinical and MR imaging variables between the groups were tested using the Student $t$ test and analysis of variance for continuous variables, the MannWhitney rank sum test and Kruskal-Wallis test for ordinal measures, and the $\chi^{2}$ test for categoric measures. The Cohen $\kappa$ agreement was used to test the reproducibility for detection of LM CE foci among the 3 raters.

The differences among the 3 assessment methods were tested using repeat analysis of variance for continuous measures and the Cochran Q for categoric measures.

Sensitivity, specificity, positive predictive value (PPV), and negative predictive value (NPV) with $95 \%$ confidence intervals were calculated among the 3 assessment methods at the patient level, using the coregistered, pre-/postcontrast 3D-FLAIR subtraction images (method C, Figure) as the criterion standard.
A nominal $P$ value of $<.05$ was considered statistically significant using 2-tailed tests.

\section{RESULTS}

\section{Demographic, Clinical, and MR Imaging Characteristics at} Baseline

Table 1 shows demographic, clinical, and MR imaging characteristics between patients with MS with or without the presence of LM CE in various disease subtypes, according to the method C classification. Patients positive for RRMS LM CE were older ( $P=$ .047), had an older age at onset $(P=.045)$, were more disabled $(P=.01)$, and had higher T2-LV $(P<.001)$ and T1-LV $(P=$ $.002)$. No differences in demographic, clinical, and MR imaging characteristics were found between patients positive and negative for SPMS and CIS LM CE.

\section{Comparison of LM CE Characteristics Using the 3 Different Assessment Methods}

On-line Table 1 shows LM CE characteristics and regional distribution, according to assessment methods A, B, or C.

In total, $51(19.8 \%)$ patients showed the presence of LM CE using method A; 39 (15.1\%), using Method B; and 39 (15.1\%), 
Table 1: Demographic, clinical, and basic MRI characteristics in patients with multiple sclerosis with and without leptomeningeal contrast enhancement, according to the method C classification

\begin{tabular}{|c|c|c|c|c|c|c|c|c|c|}
\hline \multirow[b]{2}{*}{$\begin{array}{c}\text { Demographic and } \\
\text { Clinical Characteristics }\end{array}$} & \multicolumn{3}{|c|}{$\mathrm{RR}(n=212)$} & \multicolumn{3}{|c|}{$\mathrm{SP}(n=32)$} & \multicolumn{3}{|c|}{$\operatorname{CIS}(n=14)$} \\
\hline & $\begin{array}{l}\text { Negative for } \\
\text { LM CE }(n=179)\end{array}$ & $\begin{array}{l}\text { Positive for } \\
\text { LM CE }(n=33)\end{array}$ & $\begin{array}{c}P \\
\text { Value }^{\mathrm{a}}\end{array}$ & $\begin{array}{l}\text { Negative for } \\
\text { LM CE }(n=27)\end{array}$ & $\begin{array}{l}\text { Positive for } \\
\text { LM CE }(n=5)\end{array}$ & $\begin{array}{c}P \\
\text { Value }^{\mathrm{a}}\end{array}$ & $\begin{array}{l}\text { Negative for } \\
\text { LM CE }(n=13)\end{array}$ & $\begin{array}{l}\text { Positive for } \\
\text { LM CE }(n=1)\end{array}$ & $\begin{array}{c}P \\
\text { Value }^{a}\end{array}$ \\
\hline Female (No.) (\%) & $138(77.1)$ & $23(69.7)$ & .361 & $22(81.5)$ & $4(80.0)$ & .938 & $10(76.9)$ & $0(0)$ & .286 \\
\hline Age (mean) (SD) (yr) & $46.8(12.4)$ & $51.3(9.8)$ & $.047^{\mathrm{b}}$ & $56.8(8.3)$ & $60.6(7.6)$ & .348 & $43.4(12.6)$ & $32.0(-)$ & .401 \\
\hline Age of onset (mean) (SD) (yr) & $32.3(11.5)$ & $36.6(9.8)$ & $.045^{\mathrm{b}}$ & $31.4(10.4)$ & $40.0(5.4)$ & .086 & $37.8(11.8)$ & $32.0(-)$ & .999 \\
\hline Disease duration, (mean) (SD) (yr) & $14.5(9.2)$ & $14.7(9.1)$ & .916 & $25.4(12.9)$ & $20.8(5.6)$ & .441 & $5.4(6.1)$ & $0(-)$ & .645 \\
\hline EDSS (median) (IQR) & $2.5(1.5-3.5)$ & $3.0(2.0-4.5)$ & $.010^{\mathrm{b}}$ & $6.0(6.0-7.0)$ & $6.5(3.5-7.5)$ & .658 & $1.5(1.0-2.0)$ & $1.5(1.5-1.5)$ & .999 \\
\hline \multicolumn{10}{|l|}{ DMT (No.) (\%) } \\
\hline Interferon & $38(21.3)$ & $8(24.4)$ & .220 & $3(11.1)$ & $1(20.0)$ & .845 & $6(46.2)$ & $0(0)$ & .160 \\
\hline Glatiramer acetate & 29 (16.2) & $6(18.2)$ & & $2(7.4)$ & $1(20.0)$ & & $0(0)$ & $0(0)$ & \\
\hline Natalizumab & $22(12.3)$ & $5(15.2)$ & & $1(3.7)$ & $1(20.0)$ & & $1(7.7)$ & $0(0)$ & \\
\hline Rituximab & $3(1.7)$ & $1(3.0)$ & & $0(0)$ & $0(0)$ & & $0(0)$ & $0(0)$ & \\
\hline Fingolimod & $7(3.9)$ & $5(15.2)$ & & $0(0)$ & $0(0)$ & & $0(0)$ & $0(0)$ & \\
\hline Dimethyl fumarate & $30(16.8)$ & $2(6.1)$ & & $1(3.7)$ & $0(0)$ & & $0(0)$ & $0(0)$ & \\
\hline Teriflunomide & $7(3.9)$ & $1(3.0)$ & & $6(22.2)$ & $1(20.0)$ & & $0(0)$ & $0(0)$ & \\
\hline IVIG & $6(3.3)$ & $0(0)$ & & $2(7.4)$ & $0(0)$ & & $1(7.7)$ & $0(0)$ & \\
\hline Other $\mathrm{DMT}^{\mathrm{c}}$ & $3(1.7)$ & $1(0)$ & & $1(3.7)$ & $0(0)$ & & $0(0)$ & $1(100)$ & \\
\hline Combination & $13(7.3)$ & $1(3.0)$ & & $2(7.4)$ & $0(0)$ & & $1(7.7)$ & $0(0)$ & \\
\hline No DMT & $19(10.6)$ & $4(12.1)$ & & $9(33.3)$ & $1(20.0)$ & & $4(30.8)$ & $0(0)$ & \\
\hline T2-LV (mean) (SD) & $8.86(10.42)$ & 16.62 (12.11) & $<.001^{\mathrm{b}}$ & 15.19 (17.22) & 14.38 (11.53) & .920 & $5.79(7.34)$ & $1.38(-)$ & .574 \\
\hline TI-LV (mean) (SD) & $2.55(4.82)$ & $5.65(8.25)$ & $.002^{\mathrm{b}}$ & $5.69(8.62)$ & $5.45(4.62)$ & .579 & $1.87(4.01)$ & $0.25(-)$ & .704 \\
\hline Gd-LN (mean) (SD) & $0.15(0.61)$ & $0.55(1.55)$ & .290 & $1.37(4.77)$ & $0(0)$ & .725 & $0.31(0.86)$ & $1(-)$ & .286 \\
\hline Gd-LV (mean) (SD) & $0.01(0.05)$ & $0.05(0.17)$ & .282 & $0.20(0.72)$ & $0(0)$ & .725 & $0.02(0.06)$ & $0.08(-)$ & .286 \\
\hline
\end{tabular}

Note:-LN indicates lesion number; -, indicates not available; DMT, disease modifying therapy; EDSS, Expanded Disability Status Scale; IQR, interquartile range; IVIG, intravenous immunoglobulin.

a $P$ values represent positive and negative LM CE group comparisons. The $P$ values were derived using the $\chi^{2}$ test, Fisher exact test, Student $t$ test, analysis of variance, Mann-Whitney $U$ test, and Kruskal-Wallis test.

b Significant $P$ value $<.05$.

'Other DMT therapies included intravenous methylprednisolone, intravenous immunoglobulin, mitoxantrone, and mycophenolate mofetil.

using method $\mathrm{C}(P=.002)$. There were more multiple LM CE foci detected using method $\mathrm{A}$, compared with methods $\mathrm{B}$ and $\mathrm{C}(\mathrm{P}=$ $.013)$, and the mean number of LM CE foci was the highest with method A $(P=.001)$. All LM CEs were focal, and most were detected on brain convexities (Figure and On-line Fig 1). Most of the LM CE foci were nodular, and there were no hemispheric differences. All LM CE foci were supratentorial, except 1 infratentorial foci detected with method A.

On-line Table 2 shows comparisons of patients with RRMS and SPMS according to assessment methods A, B, and C for the detection of LM CE foci. In total, 44 (20.8\%) patients with RRMS showed the presence of LM CE using method A; 35 (16.5\%), using method $\mathrm{B}$, and 33 (15.6\%), using method $\mathrm{C}(P=.004)$. The figures were 5 (15.6\%) for method A, 3 (9.4\%) for method B, and 5 (15.6\%) for method $\mathrm{C}(P=$ not significant $)$ in patients with SPMS. In patients with RRMS, there were more multiple LM CE foci detected using method A compared with methods B and C $(P=.05)$, and the mean number of LM CE foci was the highest with method A $(P=.004)$. No significant differences among the 3 methods were detected in patients with SPMS.

The mean time to analyze the 3D-FLAIR images was significantly lower with method $\mathrm{C}$, compared with methods $\mathrm{A}$ and $\mathrm{B}$ $(P<.001$, On-line Table 1$)$. In patients with both RRMS $(P<$ $.001)$ and SPMS $(P=.049)$, the mean time to analyze the $3 \mathrm{D}$ FLAIR images was significantly lower with method C, compared with methods A and B $(P<.001$, On-line Table 2$)$.

\section{Reproducibility of Leptomeningeal Contrast- Enhancement Assessment Using the 3 Different Assessment Methods}

The Cohen $\kappa$ agreement for the presence of LM CE foci (yes/no) was $0.72, P<.001$, for method A; 0.81, $P<.001$, for method $\mathrm{B}$; and $0.88, P<.001$, for method $\mathrm{C}$.

\section{A Posteriori Unblinded Concordance Analysis of the 3 Different Assessment Methods}

In total, $75 \mathrm{LM}$ CE foci were detected with method A, 55 with method $\mathrm{B}$, and 61 with method $\mathrm{C}(P=.001$, On-line Table 1$)$. Compared with the criterion standard (method C), 51 of 75 observed LM CE foci were classified as true-positive; 24, as falsepositive; 0 , as true-negative; and 10 , as false-negative using method A. This calculation yielded a sensitivity of $83.6 \%$ (95\% CI, 71.4\%-91.4\%). Compared with the criterion standard (method C), 48 of 55 observed LM CE foci were classified as true-positive; 7 , as false-positive; 17 , as true-negative; and 13 , as false-negative using method B. This yielded a sensitivity of $78.7 \%$ (95\% CI, $70 \%-87.8 \%)$ and a specificity of $70.8 \%$ (95\% CI, $48.8 \%-87.7 \%)$.

\section{Comparison of Sensitivity, Specificity, and Positive and Negative Predictive Values in Patients Using Method C as the Reference}

Table 2 shows the sensitivity, specificity, PPV, and NPV with 95\% CIs between methods A and B using method $\mathrm{C}$ as the reference in total patients with MS and according to the disease subtypes. In the total study sample, method A showed $89.8 \%$ sensitivity, $92.7 \%$ specificity, 68.6\% PPV, and 98.1\% NPV $(P<.001)$, while method B showed $84.6 \%$ sensitivity, $97.3 \%$ specificity, $84.6 \% \mathrm{PPV}$, and 97.3\% NPV $(P<.001)$. The differences in the 3 methods were significant for patients with both RRMS and SPMS, but not for CIS.

\section{DISCUSSION}

The main finding of this retrospective, observational study is that the frequency of LM CE foci is lower using the 3D-FLAIR subtraction approach compared with the postcontrast-alone approach. The 3D-FLAIR subtraction approach helped to avoid LM CE false-positive cases of patients with MS followed in a clinical 
Table 2: Sensitivity, specificity, and positive and negative predictive values in patients using method $C$ as the reference for detection of leptomeningeal contrast-enhancement foci ${ }^{a}$

\begin{tabular}{|c|c|c|c|c|c|}
\hline & Sensitivity & Specificity & PPV & NPV & $P$ Value \\
\hline \multicolumn{6}{|l|}{ Total study sample } \\
\hline Method A LM CE presence & $89.8 \%(74.8-96.7)$ & $92.7 \%(88.2-95.6)$ & $68.6 \%(53.9-80.5)$ & $98.1 \%(94.8-99.4)$ & $<.001^{\mathrm{b}}$ \\
\hline Method B LM CE presence & $84.6 \%(68.8-93.6)$ & $97.3 \%(93.8-98.8)$ & $84.6 \%(68.8-93.6)$ & $97.3 \%(93.9-98.9)$ & $<.001^{\mathrm{b}}$ \\
\hline \multicolumn{6}{|l|}{ CIS-only sample } \\
\hline Method A LM CE presence & $100 \%(5.5-100)$ & $82.3 \%(62.1-99.6)$ & $50 \%(2.7-97.3)$ & $100 \%(69.9-100)$ & .143 \\
\hline Method B LM CE presence & $100 \%(5.5-100)$ & $100 \%(71.6-100)$ & $100 \%(54.7-97.2)$ & $100 \%(71.6-100)$ & .071 \\
\hline \multicolumn{6}{|l|}{ RR-only sample } \\
\hline Method A LM CE presence & $90.1 \%(74.5-97.6)$ & $92.2 \%(86.9-95.5)$ & $68.2 \%(52.3-80.9)$ & $98.2 \%(94.5-99.5)$ & $<.001^{\mathrm{b}}$ \\
\hline Method B LM CE presence & $87.9 \%(70.9-96.0)$ & $96.7 \%(92.5-98.6)$ & $82.9 \%(65.7-92.8)$ & $97.7 \%(93.9-99.3)$ & $<.001^{\mathrm{b}}$ \\
\hline \multicolumn{6}{|l|}{ SP-only sample } \\
\hline Method A LM CE presence & $96.3 \%(79.1-99.8)$ & $96.3 \%(79.1-99.8)$ & $80 \%(29.9-98.9)$ & $96.3 \%(79.1-99.8)$ & $<.001^{\mathrm{b}}$ \\
\hline Method B LM CE presence & $60 \%(17.0-92.7)$ & $100 \%(84.9-100)$ & $100 \%(30.9-100)$ & $93.1 \%$ (75.7-98.7) & .002 \\
\hline
\end{tabular}

${ }^{a}$ Method A assessment was performed using postcontrast 3D-FLAIR images in native space; method B, using pre- and postcontrast 3D-FLAIR images in native space; and method C, using the coregistered, pre-/postcontrast 3D-FLAIR subtracted images. Data are presented as sensitivity, specificity, PPV, and NPV. In parenthesis are 95\% confidence intervals. $P$ Value refers to the significance of the contingency table (Fisher exact test).

b Significant.

routine. No LM CE frequency differences were found between patients with RRMS and SPMS followed in the clinical routine.

The reproducibility of LM CE foci assessment was the highest using the pre-/postcontrast subtraction approach. There was also a shorter time needed for LM CE foci analysis using 3D-FLAIR pre-/postcontrast subtraction images, which may lead to a more widespread adoption of this new imaging biomarker in MS clinical routine.

The true prevalence of LM CE in patients with CIS and MS is not well-established. In one of the first studies of LM CE in MS that included 299 patients with MS, ${ }^{9} 25 \%$ of patients with MS presented with LM CE, while in another study of 50 patients with MS, the prevalence was $50 \% .{ }^{10}$ Both of those studies used 3T MR imaging and high-resolution 3D-FLAIR imaging acquired at least 10 minutes after contrast injection. Another study ${ }^{14}$ that examined 29 patients with MS on 7T MR imaging using high-resolution 3D-FLAIR acquired approximately 20 minutes postcontrast injection showed 90\% LM CE prevalence. The only MS study that showed $<1 \%$ prevalence used 3T MR imaging with low-resolution 2D-FLAIR images acquired approximately 10 minutes postinjection. ${ }^{13}$

The current study is one of the first studies in the clinical routine in which 258 consecutive patients with MS were enrolled to undergo pre- and postcontrast 3D-FLAIR imaging, coregistered and subtracted. The sensitivity, specificity, PPV, and NPV among the 3 assessment LM CE methods were different at the patient level. The subtraction approach was particularly useful in depicting false-positive and false-negative LM CE foci due to various reasons such as partial voluming of brain parenchyma and blood vessels (On-line Fig 2). The a posteriori unblinded concordance analysis of the 3 different assessment methods found 24 false-positive and 10 false-negative LM CE foci using the postcontrast-alone approach, and 7 false-positive, 17 true-negative, and 13 false-negative foci using the pre- and postcontrast approach. The false-positive LM CE foci characteristics included focal aspect and nodular shape and were located predominantly in the supratentorial sulci of the frontal and temporal lobes. Therefore, we recommend using the subtraction approach for easier detection of LM CE mimics, such as meningeal blood vessels, large subarachnoid veins, and high signal intensity areas in regions adja- cent to dural venous sinuses and basal meninges related to CSF enhancement. ${ }^{9,10,12}$

In the present study of consecutive clinical routine patients with MS, we found that LM CE frequency was substantially lower compared with that in the previous studies. $9,10,12,14$ With the pre-/postcontrast 3D-FLAIR subtraction approach, the LM CE frequency was $15.6 \%$ in patients with RRMS and $15.6 \%$ in those with SPMS, and we found only 1 patient with CIS presenting with LM CE foci. Consequently, we did not confirm findings from previous studies indicating that LM CE prevalence is significantly increased in patients with SPMS compared with those with RRMS. ${ }^{9,10}$ Therefore, the frequency of LM CE foci may be substantially lower in clinical routine in patients with MS imaged on a $3 \mathrm{~T}$ scanner using a pre-/postcontrast 3D-FLAIR subtraction approach.

On modern 1.5T and 3T scanners, the 3D-FLAIR acquisition takes approximately 5-7 minutes. Adding postcontrast 3DFLAIR for the detection of LM CE increases the acquisition time in a clinical routine. The current Consortium of Multiple Sclerosis Centers MR imaging protocol guidelines include the $3 \mathrm{D}$ FLAIR precontrast examination in core recommended sequences, ${ }^{19}$ because it has been shown that 3D-FLAIR detects significantly more lesions in patients with MS compared with 2D T2-based pulse sequences, especially in the infratentorial and cortical regions. ${ }^{15,20,21}$

If the detection of LM CE foci is to become part of the clinical routine MR imaging reading assessment in MS, it should be done in the least amount of time possible and in the most accurate way. The subtraction approach had an approximately 18\% shorter time for detection of LM CE foci with respect to the postcontrastonly approach and $31 \%$ shorter time compared with pre- and postcontrast-only approach, with an average of slightly above 2 minutes used per examination, including inspection of the source images. Patients with MS without LM CE foci could be identified in $<1$ minute using the subtraction approach without the use of the source images. We believe this is a reasonable amount of time to add to the reading burden of expert MS neuroimagers with high reproducibility among the raters. Most important, our findings suggest that the subtraction approach could be sufficiently accurate for fast screening of LM CE in clinical routine in patients 
with MS. The allocated time measured just the reading component of pre-/postcontrast 3D-FLAIR subtraction images in all 3 orthogonal planes and did not take into account the running of the algorithm for creation of the images themselves. However, we anticipate that the algorithm applied in this study can be run fully automatically at the MR imaging scanner workstation or a console in $<1$ minute, producing corresponding per-slice images.

The strength of the study is the use of a large cohort of patients with MS consecutively enrolled in a clinical routine and a careful methodologic approach for the detection of LM CE foci. The limitation of the study includes lack of longitudinal serial imaging in these patients with MS, which is underway in our center. In addition, the subtraction approach for detection of LM CE should be also validated using cases without MS and independent MS cohorts in future studies.

\section{CONCLUSIONS}

The frequency of LM CE foci is lower using the pre-/post-3DFLAIR subtraction approach, compared with the postcontrast alone approach. No LM CE frequency differences were found between patients with RRMS and SPMS in a clinical routine. Use of subtraction imaging helped to avoid false-positive cases, decreased reading time, and increased the accuracy of LM CE foci detection in a clinical routine.

Disclosures: Robert Zivadinov-UNRELATED: Consultancy: Sanofi Genzyme, Novartis, EMD Serono; Grants/Grants Pending: Sanofi Genzyme, Novartis, IMS Health*; Payment for Lectures Including Service on Speakers Bureaus: Sanofi Genzyme, Novartis, EMD Serono. Michael G. Dwyer-UNRELATED: Consultancy: Claret Medical; Grants/Grants Pending: Novartis.* Bianca Weinstock-Guttman-UNRELATED: Board Membership: Biogen Idec, Teva Pharmaceutical Industries, EMD Serono, Sanofi Genzyme, Genentech, Novartis; Consultancy: Biogen Idec, Teva Pharmaceutical Industries, EMD Serono, Sanofi Genzyme, Genentech, Novartis; Grants/Grants Pending: Biogen Idec, Teva Pharmaceutical Industries, EMD Serono, Sanofi Genzyme, Genentech, Novartis*; Payment for Lectures Including Service on Speakers Bureaus: Biogen, Teva Pharmaceutical Industries, Novartis, Genentech. David Hojnacki—UNRELATED: Consultancy: Biogen, Teva Pharmaceutical Industries, Genentech, Serono; Payment for Lectures Including Service on Speakers Bureaus: Biogen, Teva Pharmaceutical Industries, Genentech, Serono. *Money paid to the institution.

\section{REFERENCES}

1. Popescu BF, Lucchinetti CF. Meningeal and cortical grey matter pathology in multiple sclerosis. BMC Neurol 2012;12:11 CrossRef Medline

2. Magliozzi R, Howell O, Vora A, et al. Meningeal B-cell follicles in secondary progressive multiple sclerosis associate with early onset of disease and severe cortical pathology. Brain 2007;130:1089-104 Medline

3. Magliozzi R, Howell OW, Reeves $\mathrm{C}$, et al. A gradient of neuronal loss and meningeal inflammation in multiple sclerosis. Ann Neurol 2010;68:477-93 CrossRef Medline

4. Serafini B, Rosicarelli B, Magliozzi R, et al. Detection of ectopic B-cell follicles with germinal centers in the meninges of patients with secondary progressive multiple sclerosis. Brain Pathol 2004;14:164-74 CrossRef Medline
5. Lucchinetti CF, Popescu BF, Bunyan RF, et al. Inflammatory cortical demyelination in early multiple sclerosis. $N$ Engl J Med 2011;365: 2188-97 CrossRef Medline

6. Choi SR, Howell OW, Carassiti D, et al. Meningeal inflammation plays a role in the pathology of primary progressive multiple sclerosis. Brain 2012;135:2925-37 CrossRef Medline

7. Howell OW, Reeves CA, Nicholas R, et al. Meningeal inflammation is widespread and linked to cortical pathology in multiple sclerosis. Brain 2011;134:2755-71 CrossRef Medline

8. Zurawski J, Lassmann H, Bakshi R. Use of magnetic resonance imaging to visualize leptomeningeal inflammation in patients with multiple sclerosis: a review. JAMA Neurol 2017;74:100-09 CrossRef Medline

9. Absinta M, Vuolo L, Rao A, et al. Gadolinium-based MRI characterization of leptomeningeal inflammation in multiple sclerosis. $\mathrm{Neu}$ rology 2015;85:18-28 CrossRef Medline

10. Zivadinov R, Ramasamy DP, Vaneckova M, et al. Leptomeningeal contrast enhancement is associated with progression of cortical atrophy in MS: a retrospective, pilot, observational longitudinal study. Mult Scler 2017;23:1336-45 CrossRef Medline

11. Xia Z, Steele SU, Bakshi A, et al. Assessment of early evidence of multiple sclerosis in a prospective study of asymptomatic high-risk family members. JAMA Neurol 2017;74:293-300 CrossRef Medline

12. Absinta M, Cortese IC, Vuolo L, et al. Leptomeningeal gadolinium enhancement across the spectrum of chronic neuroinflammatory diseases. Neurology 2017;88:1439-44 CrossRef Medline

13. Eisele P, Griebe M, Szabo K, et al. Investigation of leptomeningeal enhancement in MS: a postcontrast FLAIR MRI study. Neurology 2015;84:770-75 CrossRef Medline

14. Harrison DM, Wang KY, Fiol J, et al. Leptomeningeal enhancement at $7 \mathrm{~T}$ in multiple sclerosis: frequency, morphology, and relationship to cortical volume. J Neuroimaging 2017;27:461-68 CrossRef Medline

15. Polak P, Magnano C, Zivadinov R, et al. 3D FLAIRED: 3D fluid attenuated inversion recovery for enhanced detection of lesions in multiple sclerosis. Magn Reson Med 2012;68:874-81 CrossRef Medline

16. Busse RF, Hariharan $\mathrm{H}, \mathrm{Vu}$ A, et al. Fast spin echo sequences with very long echo trains: design of variable refocusing flip angle schedules and generation of clinical T2 contrast. Magn Reson Med 2006; 55:1030-37 CrossRef Medline

17. Jenkinson M, Bannister P, Brady M, et al. Improved optimization for the robust and accurate linear registration and motion correction of brain images. Neuroimage 2002;17:825-41 CrossRef Medline

18. Zivadinov R, Heininen-Brown M, Schirda CV, et al. Abnormal subcortical deep-gray matter susceptibility-weighted imaging filtered phase measurements in patients with multiple sclerosis: a case-control study. Neuroimage 2012;59:331-39 CrossRef Medline

19. Traboulsee A, Simon JH, Stone L, et al. Revised Recommendations of the Consortium of MS Centers Task Force for a Standardized MRI Protocol and Clinical Guidelines for the Diagnosis and Follow-Up of Multiple Sclerosis. AJNR Am J Neuroradiol 2016;37:394-401 CrossRef Medline

20. Bink A, Schmitt M, Gaa J, et al. Detection of lesions in multiple sclerosis by 2D FLAIR and single-slab 3D FLAIR sequences at $3.0 \mathrm{~T}$ : initial results. Eur Radiol 2006;16:1104-10 CrossRef Medline

21. Moraal B, Roosendaal SD, Pouwels PJ, et al. Multi-contrast, isotropic, single-slab 3D MR imaging in multiple sclerosis. Eur Radiol 2008;18:2311-20 CrossRef Medline 